\title{
Oscillations, traveling fronts and patterns in a supramolecular system
}

Jorge Leira-Iglesias ${ }^{1}$, Alessandra Tassoni ${ }^{1}$, Takuji Adachi ${ }^{1}$, Michael Stich ${ }^{2}$ and Thomas M. Hermans $^{1 *}$

${ }^{1}$ University of Strasbourg, CNRS, ISIS UMR 7006, F-67000 Strasbourg, France. ${ }^{2}$ Non-linearity and Complexity Research Group, Systems Analytics Research Institute, Engineering and Applied Science, Aston Triangle, Birmingham B4 7ET, United Kingdom. ${ }^{*}$ e-mail: hermans@unistra.fr 
Supplementary Information

\section{Oscillations, traveling fronts and patterns in a supramolecular system}

Jorge Leira-Iglesias ${ }^{1}$, Alessandra Tassoni ${ }^{1}$, Takuji Adachi ${ }^{1}$, Michael Stich ${ }^{2}$ and Thomas M. Hermans $^{1 *}$

Corresponding author: * hermans@unistra.fr

Contents:

Supplementary Section 1. UV-Vis and light scattering experiments

Supplementary Section 2. Cooperative polymerization mechanism

Supplementary Section 3. One redox cycle

Supplementary Section 4. Supramolecular oscillator

Supplementary Section 5. Supramolecular oscillator model

Supplementary Section 6. Supramolecular fronts and patterns

Supplementary Section 7. Captions for videos

Supplementary References 


\section{Supplementary Section 1. UV-Vis and light scattering experiments}

UV-Vis experiments showing the growth of $\mathbf{P D I}_{\text {mon }}$ to $\mathbf{P D I}_{\text {assem }}$ (i.e., black to red spectra in Supplementary Fig. 1). ${ }^{1}$ A new band appears at $528 \mathrm{~nm}$ having a sigmoidal time progression indicating that PDI assembles via a cooperative polymerization mechanism (see section 2 below for a more detailed analysis). UV-Vis spectra are shown as optical density $O D$ since there is contribution of scattering as well as absorption. We assume that the optical density is directly proportional to the degree of aggregation $\alpha$ and is experimentally determined as: $[O D]_{528 \mathrm{~nm}}=$ $\alpha[O D]_{P D I_{\text {assem }, 528 n m}}+(1-\alpha)[O D]_{P D I_{m o n, 528 n m}} \cdot{ }^{2}$ Neutral PDI $_{\text {assem }}$ structures are $\sim 2 \mu \mathrm{m}$ in size, whereas reduced PDI $^{--}$and PDI $^{2-}$ are only $~ 100 ~ n m$ at $100 \mu \mathrm{M}$ (Supplementary Fig. 2). Dynamic and static light scattering show the size and scattering progression of the PDI mon cooperative growth (Supplementary Fig. 3).

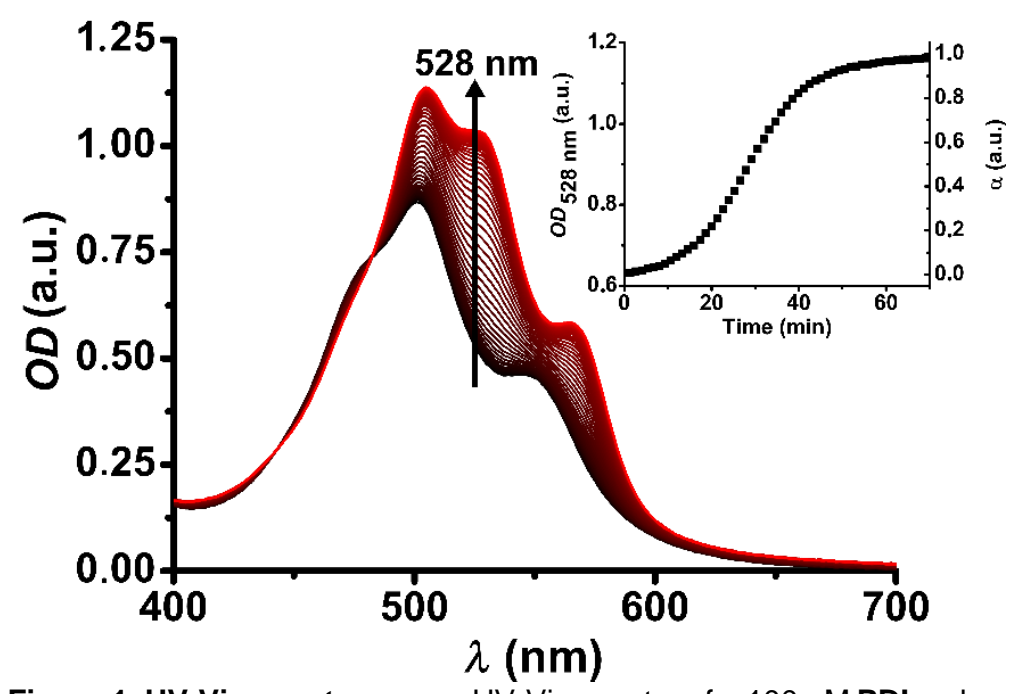

Supplementary Figure 1. UV-Vis spectroscopy. UV-Vis spectra of a $100 \mu \mathrm{M}$ PDImon borate buffer solution (PDI solution was reduced to PDI ${ }^{2-}$ and spontaneously oxidized back to neutral PDI, see Methods) at 500 $\mathrm{rpm}$. The inset shows the time progression of the $528 \mathrm{~nm}$ band which can be used to track the cooperative polymerization.

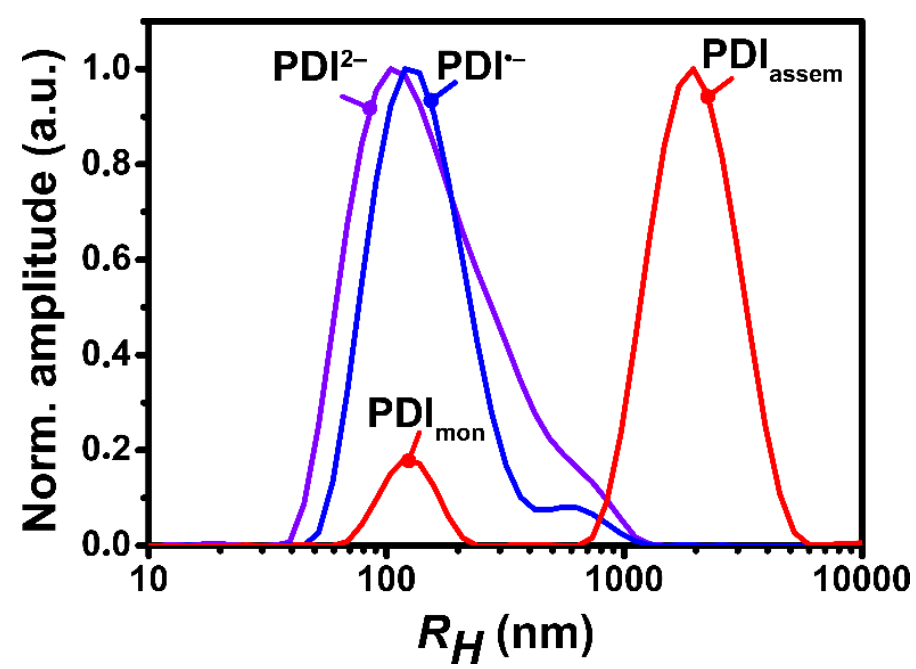

Supplementary Figure 2. Dynamic (visible, $639 \mathrm{~nm}$ ) light scattering measurements. Size distributions are shown for PDI (red curve), PDI'- (blue curve), and PDI ${ }^{2-}$ (purple curve) at $100 \mu \mathrm{M}$ in borate buffer solution. 


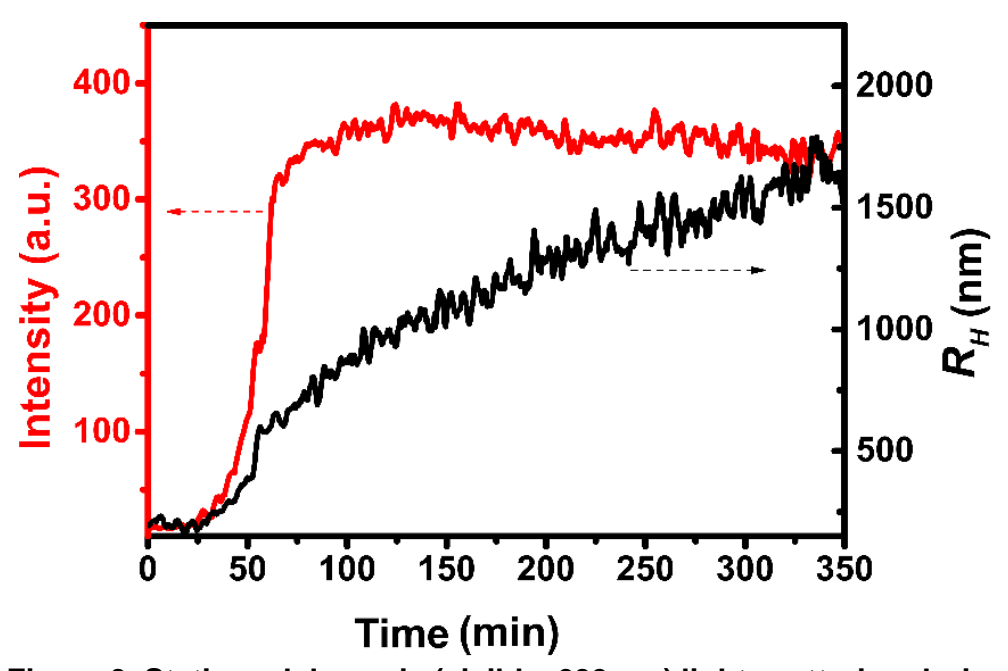

Supplementary Figure 3. Static and dynamic (visible, $639 \mathrm{~nm}$ ) light scattering during the progression of $\mathrm{PDI}^{2-}$ to $\mathrm{PDI}_{\text {mon }}$ to $\mathrm{PDI}_{\text {assem. }}$ Hydrodynamic radius (black, right axis) from dynamic light scattering, and static light scattering intensity (red, left axis) vs time of supramolecular polymerization at $100 \mu \mathrm{M}$ (non-stirred, since we cannot do DLS while stirring). The evolution of the size vs time has a sigmoidal time progression in agreement with observations by UV-Vis spectroscopy (cf. Supplementary Figure 1). PDI ${ }^{2-}$ absorbs at 639 $\mathrm{nm}$, which decreases the total scattered intensity. When $\mathbf{P D I}^{2-}$ disappears due to oxidation, a large jump in static light scattering intensity can be seen ( $\sim 50 \mathrm{~min}$ ). Therefore, visible static light scattering cannot be used quantitatively. The intensity autocorrelation functions for dynamic light scattering were fitted with a monoexponential decay function $g_{1}(t)=e^{-\Gamma t}$. 


\section{Supplementary Section 2. Cooperative polymerization mechanism}

PDI $_{\text {mon }}$ assembles via a cooperative polymerization mechanism into $\mathbf{P D I}_{\text {assem }}$ structures. ${ }^{1}$ We determined the half times (i.e., the time needed for half of the PDI molecules to be assembled) at different concentrations and used them to discriminate between various available models. ${ }^{3} \mathrm{~A}$ scaling factor $\gamma$ can be obtained from the plot of the half times vs. concentration (Supplementary Fig. 4). The scaling factor $\gamma$ is $0.485 \pm 0.036$ which indicates that PDI polymerization involves more processes than simple nucleation and elongation steps. In addition, the value of $\gamma$ suggests that fragmentation is also involved in the polymerization mechanism. ${ }^{3}$ The data were analysed using three different analytical models (using the AmyloFit platform) ${ }^{3}$ : nucleation-elongation, nucleation-elongation including fragmentation, and saturating elongation + fragmentation (see Supplementary Fig. 5 and Fig. $1 \mathrm{c}$ in the main text). The fitting improves when the fragmentation step is included into the model judged from the mean residual error (MRE), when compared to simple nucleation-elongation. The saturating elongation and fragmentation model gives the best fitting (i.e., lowest mean residual error, Supplementary Fig. 6) which indicates that the elongation involves multiple steps and thus, PDI polymerization is more complex than simple nucleationelongation. In addition, nucleus size 2 gives the best fitting for all models (Supplementary Fig. 6).

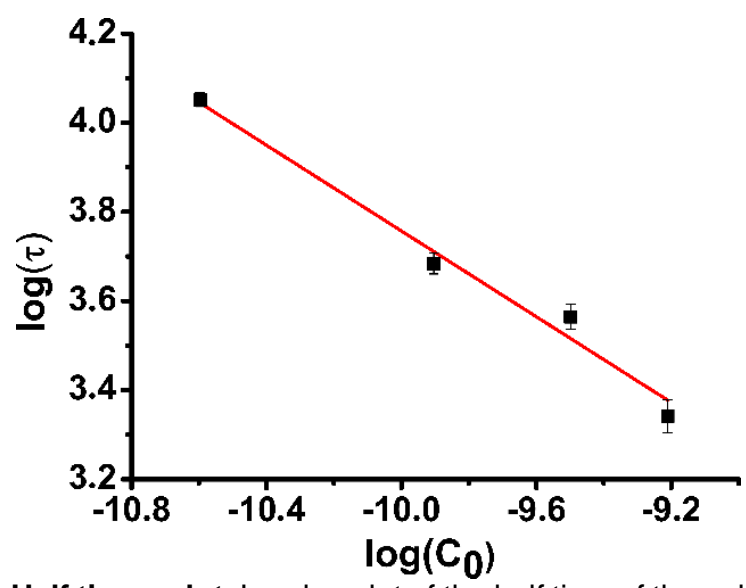

Supplementary Figure 4. Half times plot. Log-log plot of the half time of the polymerization at different PDI concentrations (i.e., 100, 75, 50 and $25 \mu \mathrm{M}$ ). The scaling exponent $\gamma$ was obtained by fitting the following equation: $\log (\tau)=\gamma \cdot \log \left(C_{0}\right)+k$. The slope yields the scaling exponent $\gamma=-0.485 \pm 0.036$.
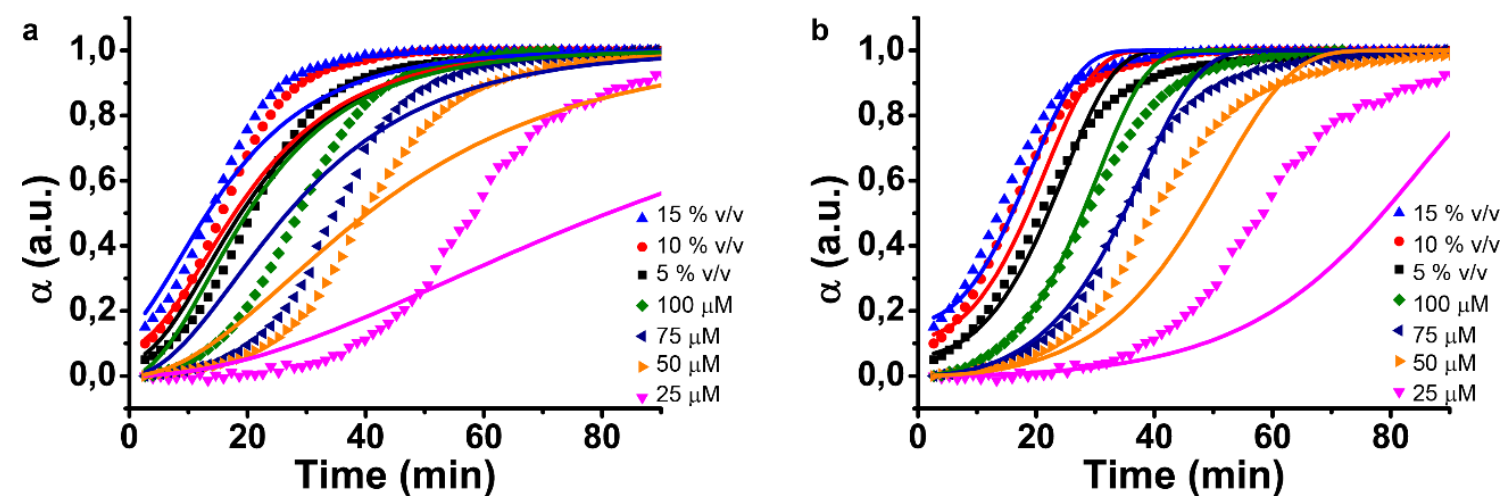

Supplementary Figure 5. Alternative model fits of PDI supramolecular polymerization. PDI supramolecular polymerization of the different PDI concentrations at $1000 \mathrm{rpm}$ (degree of aggregation $\alpha$ indicates the growth of PDI assemblies). Seeding experiments (15, 10 and $5 \%$ in $\mathrm{v} / \mathrm{v}$ of $1 \mathrm{~h}$ PDImon aged solution, i.e., seeds were added to a freshly oxidized PDImon solution) were performed on $100 \mu \mathrm{M}$ PDImon borate buffer solution at the same stirring rate (i.e., $1000 \mathrm{rpm}$ ). Afterwards, the data was analysed with a, a simple nucleation-elongation model that gives $k_{n u c}=2.6 \mathrm{M}^{-1} \cdot \mathrm{min}^{-1}, k_{e l}=1.1 \cdot 10^{5} \mathrm{M}^{-1} \cdot \mathrm{min}^{-1}, k_{r e l}=3.6 \cdot 10^{-13} \mathrm{~min}^{-}$ $1, n=2$ and mean residual error (MRE) $=0.016$. $\mathbf{b}$, a nucleation-elongation model that includes fragmentation that gives $k_{\text {nuc }}=26 \mathrm{M}^{-1} \cdot \mathrm{min}^{-1}, k_{e l}=1.8 \cdot 10^{3} \mathrm{M}^{-1} \cdot \mathrm{min}^{-1}, k_{\text {rel }}=1 \cdot 10^{-15} \mathrm{~min}^{-1}, k_{\text {frag }}=0.048 \mathrm{~min}^{-1}, \mathrm{n}=2$ and MRE $=$ 0.0058 . 


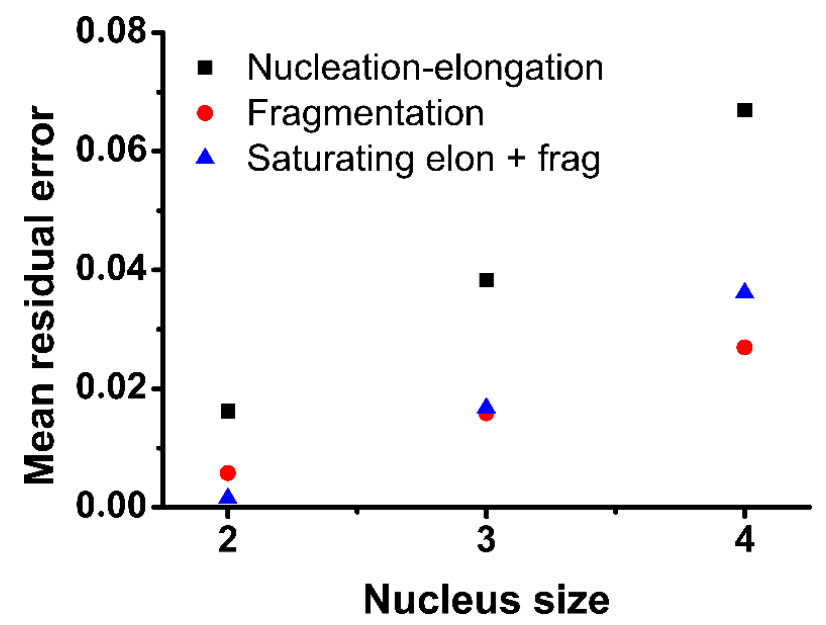

Supplementary Figure 6. Effect of the nucleus size on the error of fitting the polymerization mechanism. Mean residual error of the global fitting of the nucleation-elongation (black squares), nucleation-elongation including fragmentation (red dots) and saturating elongation + fragmentation models (blue triangles) vs nucleus size.

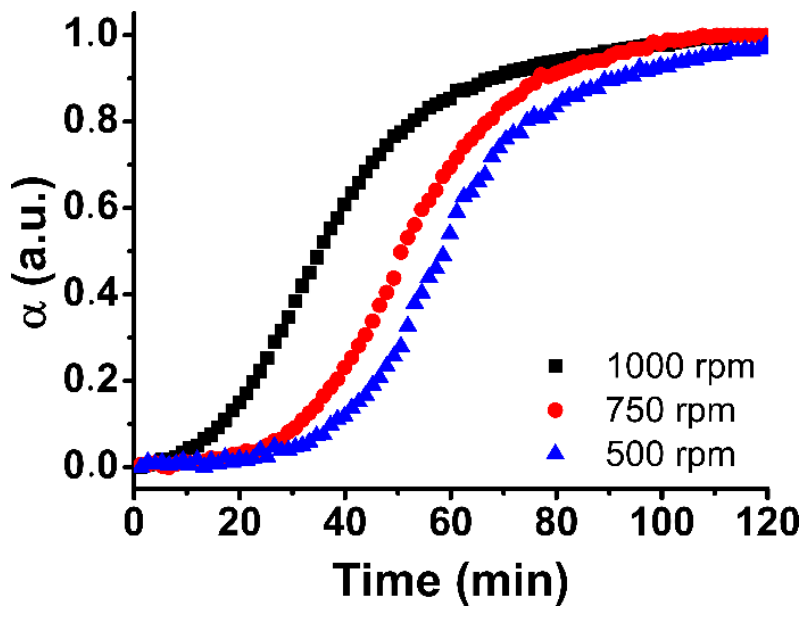

Supplementary Figure 7. Cooperative polymerization of PDI at different stirring rates. $2 \mathrm{~mL}$ of a 100 $\mu \mathrm{M}$ PDI borate buffer solution was reduced to $\mathrm{PDI}^{2-}$ upon addition of $2 \mu \mathrm{L}$ of $400 \mathrm{mM} \mathrm{Na}_{2} \mathrm{~S}_{2} \mathrm{O}_{4}$ solution. After spontaneous oxidation in air, the cooperative growth of PDImon was followed at different stirring rates $(1000$, 750 and $500 \mathrm{rpm})$. 


\section{Supplementary Section 3. One redox cycle}

A colour CCD camera permits to track separately red, blue and green colour channels of a PDI solution. Combined with a fast diode-array spectrophotometer, it can be used to determine the relative concentration of the different species present in solution. Prior to reducing the system, the starting values of red and blue channel and the diode-array (i.e., black line in the inset, Supplementary Fig.8) confirm the presence of as prepared PDI assemblies. The intensity of the red channel drops quickly (i.e., intensity goes from $\sim 80$ to $\sim 40$ ) upon addition of reductant $\mathrm{Na}_{2} \mathrm{~S}_{2} \mathrm{O}_{4}$. The diode-array (i.e., purple dash-dot line in the inset, Supplementary Figure 8) confirms the formation of $\mathbf{P D I}^{2-}$. PDI mon is reached after spontaneous oxidation in the air. The blue channel intensity increases and the red channel decreases indicating the transient formation of PDI-- (the spectrum shows the characteristic bands of PDI-- at 727 and $813 \mathrm{~nm}$, blue dashed spectrum in the inset of Supplementary Fig. 8). Afterwards, the red channel intensity quickly increases and the blue channel intensity decreases showing the presence of PDI mon $_{\text {(pink dotted }}$ spectrum in the inset). In addition, a slow sigmoidal increase in both channels indicates the

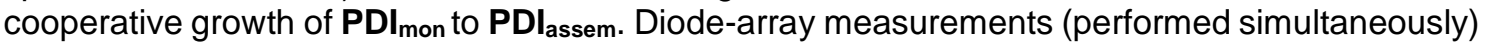
confirmed the presence of $\mathbf{P D I}_{\text {assem }}$ (green dotted spectrum in the inset). In short, the colour camera and diode-array UV-Vis detector permit to track all the species present in a full redox cycle PDI $\rightarrow$ PDI $^{2-} \rightarrow$ PDI $^{--} \rightarrow$ PDI $_{\text {mon }} \rightarrow$ PDI $_{\text {assem }}$.

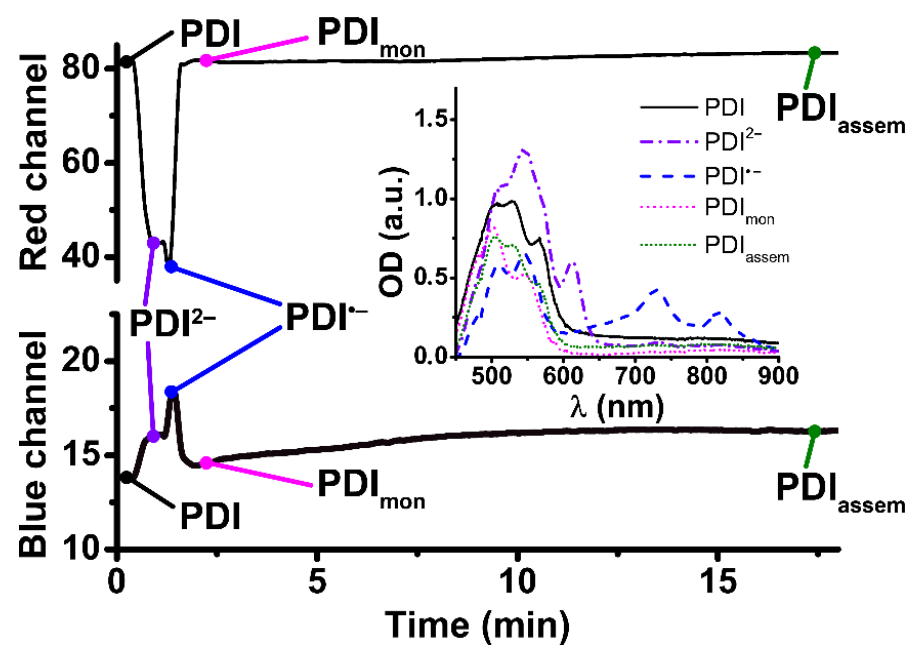

Supplementary Figure 8. One redox cycle. The system was measured in a semi-batch reactor at 1000 rpm with a CCD camera and diode-array UV-Vis detector. $2.25 \mathrm{~mL}$ of a $25 \mu \mathrm{M}$ PDI borate buffer solution is reduced to PDI $^{2-}$ upon the addition of $2 \mu \mathrm{L}$ of $400 \mathrm{mM} \mathrm{Na}_{2} \mathrm{~S}_{2} \mathrm{O}_{4}$ solution. Afterwards, PDI ${ }^{2-}$ was oxidized back to $\mathbf{P D I}_{\text {mon }}$ via $\mathbf{P D I} \mathbf{P}^{-}$formation. PDI mon grows via a cooperative polymerization mechanism leading to

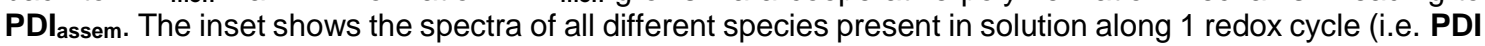
$\left.\rightarrow \mathrm{PDI}^{2-} \rightarrow \mathrm{PDI}^{--} \rightarrow \mathrm{PDI}_{\text {mon }} \rightarrow \mathrm{PDl}_{\text {assem }}\right)$.
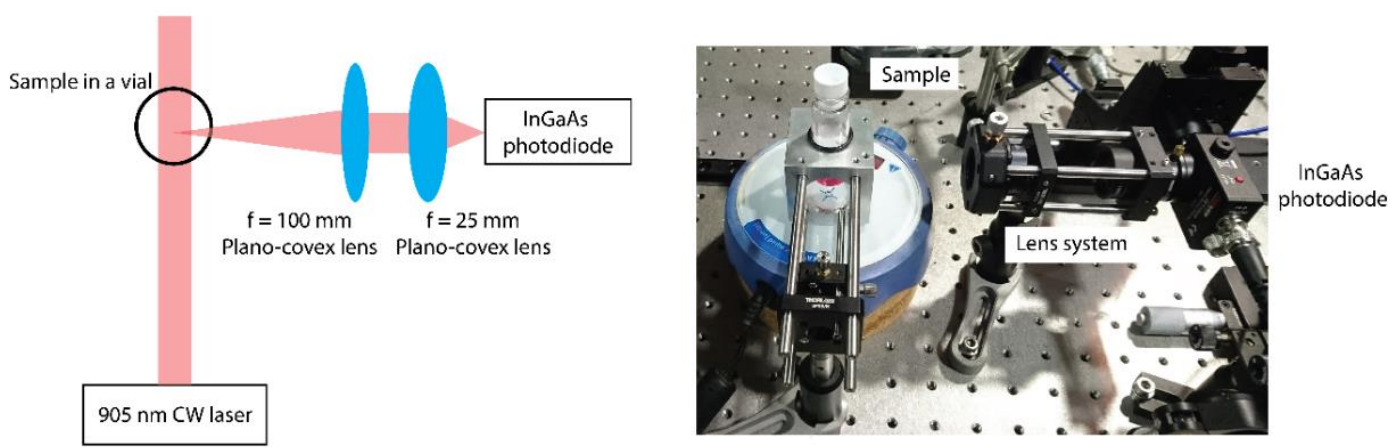

Supplementary Figure 9. Scheme of the near-infra red light scattering setup using a $905 \mathrm{~nm}$ laser. The scattering intensity was recorded using a home-built setup (photo shown on the right). This wavelength was selected because none of the three species (PDI, PDI'- or $\mathbf{P D I}^{2-}$ ) have light absorption at $905 \mathrm{~nm}$ (cf. Supplementary Figure 8). 

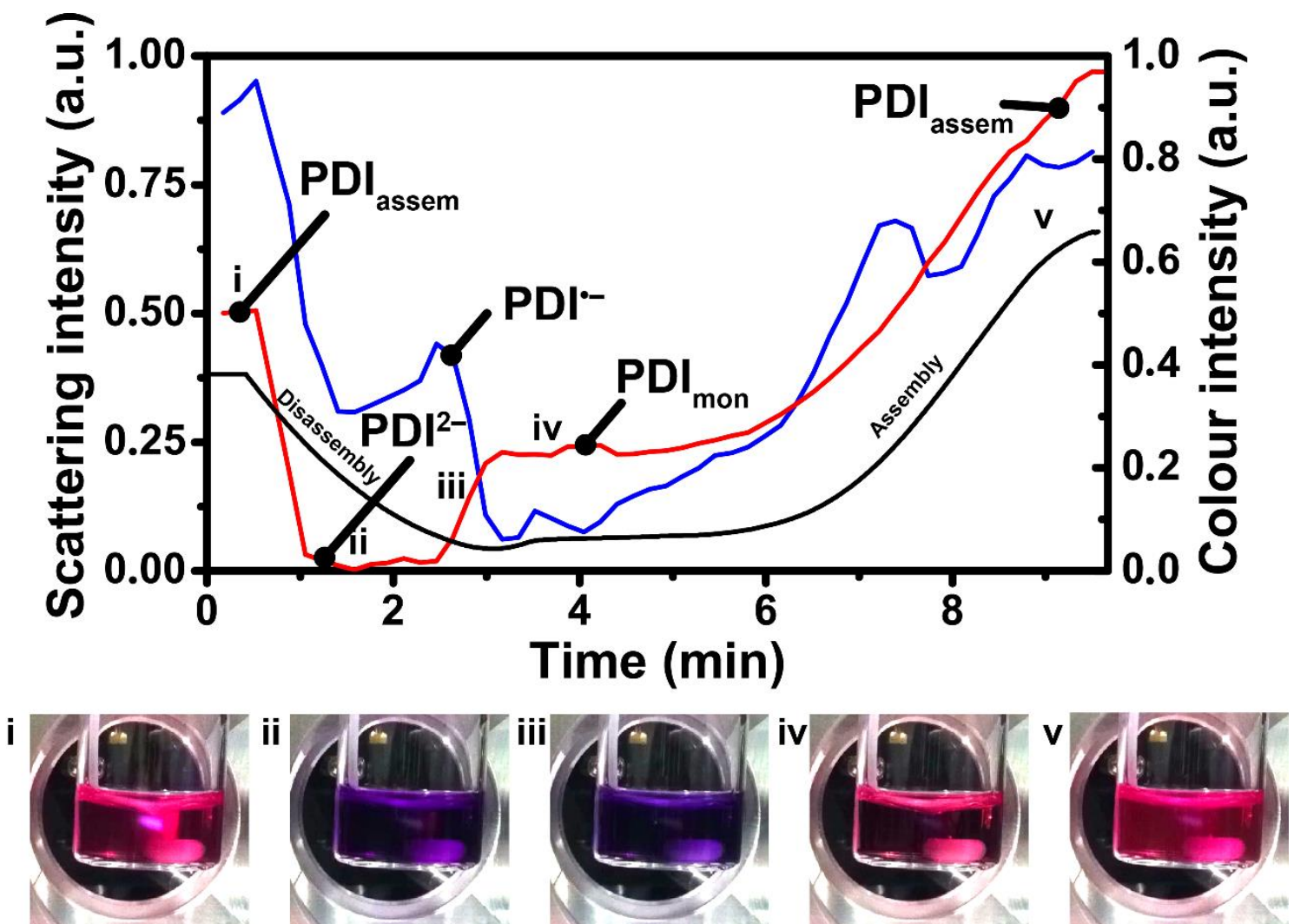

Supplementary Figure 10. Near-infra red light scattering intensity and colour tracking of PDI species during one redox cycle. The system was measured in a semi-batch reactor at $1000 \mathrm{rpm}$ using a $905 \mathrm{~nm}$ laser (cf. Supplementary Figure 9) and a camera. PDI (i at $t=0 \mathrm{~min}, 2.25 \mathrm{~mL}$ of a $25 \mu \mathrm{M}$ solution) is reduced to $\mathbf{P D I}^{2-}$ upon the addition of the reductant (ii, $t=1.2 \mathrm{~min}$ ) inducing a decrease in the scattering intensity (black curve, left axis) and in the red and blue channel intensity (red and blue curves on the right axis, respectively). Afterwards, $\mathbf{P D I}^{2-}$ was oxidized back to $\mathbf{P D I}_{\operatorname{mon}}$ (iv, $t=4 \mathrm{~min}$ ) via $\mathbf{P D I}^{--}$formation (iii, $t=2.75$ $\mathrm{min}$ ), while reaching a minimum in the scattering intensity and a maximum in the blue channel. PDImon grows via a cooperative polymerization mechanism leading to $\mathrm{PDl}_{\text {assem }}(\mathrm{v}, t=9 \mathrm{~min})$ where an increase of the scattering intensity and the red/blue channel can be observed. 

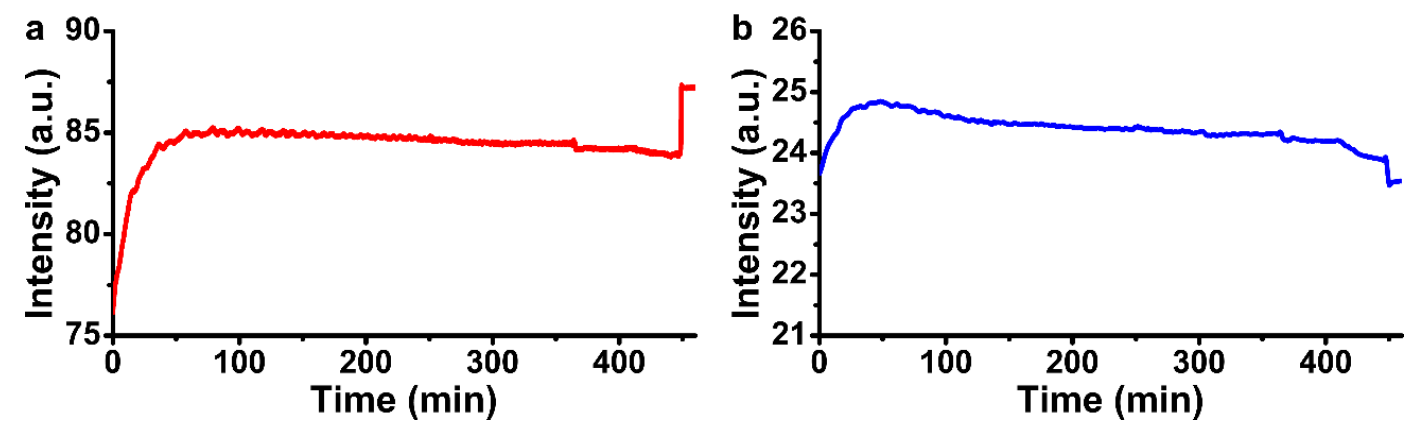

Supplementary Figure 11. Steady state 'SS'. $2.25 \mathrm{~mL}$ of a $100 \mu \mathrm{M}$ PDI borate buffer solution was reduced to $\mathbf{P D I}^{2-}$ by adding $2 \mu \mathrm{L}$ of a $400 \mathrm{mM} \mathrm{Na}_{2} \mathrm{~S}_{2} \mathrm{O}_{4}$ solution. Constant inflow of reductant $(0.7 \mu \mathrm{L} / \mathrm{min})$ was set once PDImon was present in solution. After $450 \mathrm{~min}$, inflow of chemical fuel was stopped leading to a, a minor increase of the intensity of the red channel, $\mathbf{b}$, a minor decrease of the intensity of the blue channel and thus, full oxidation to PDlassem.

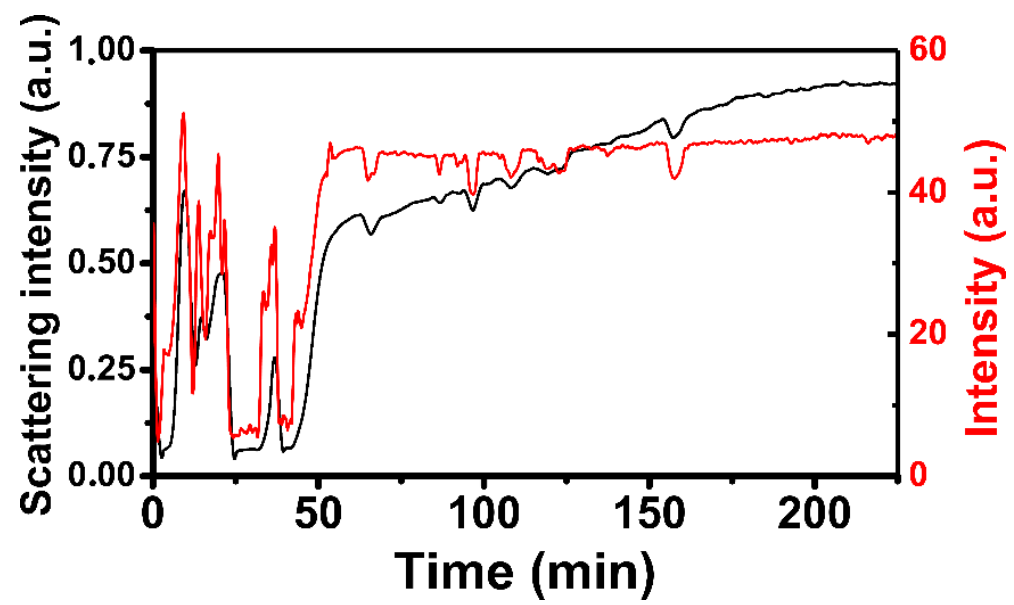

Supplementary Figure 12. Near-infra red light scattering and colour tracking during supramolecular oscillations. The scattering intensity (left axis, black line) and the red channel colour intensity (right axis, red line) of a $75 \mu \mathrm{M}$ PDI solution are tracked during oscillations in a semi-batch reactor at $1000 \mathrm{rpm}$ using a $905 \mathrm{~nm}$ laser line and a camera (see methods). 

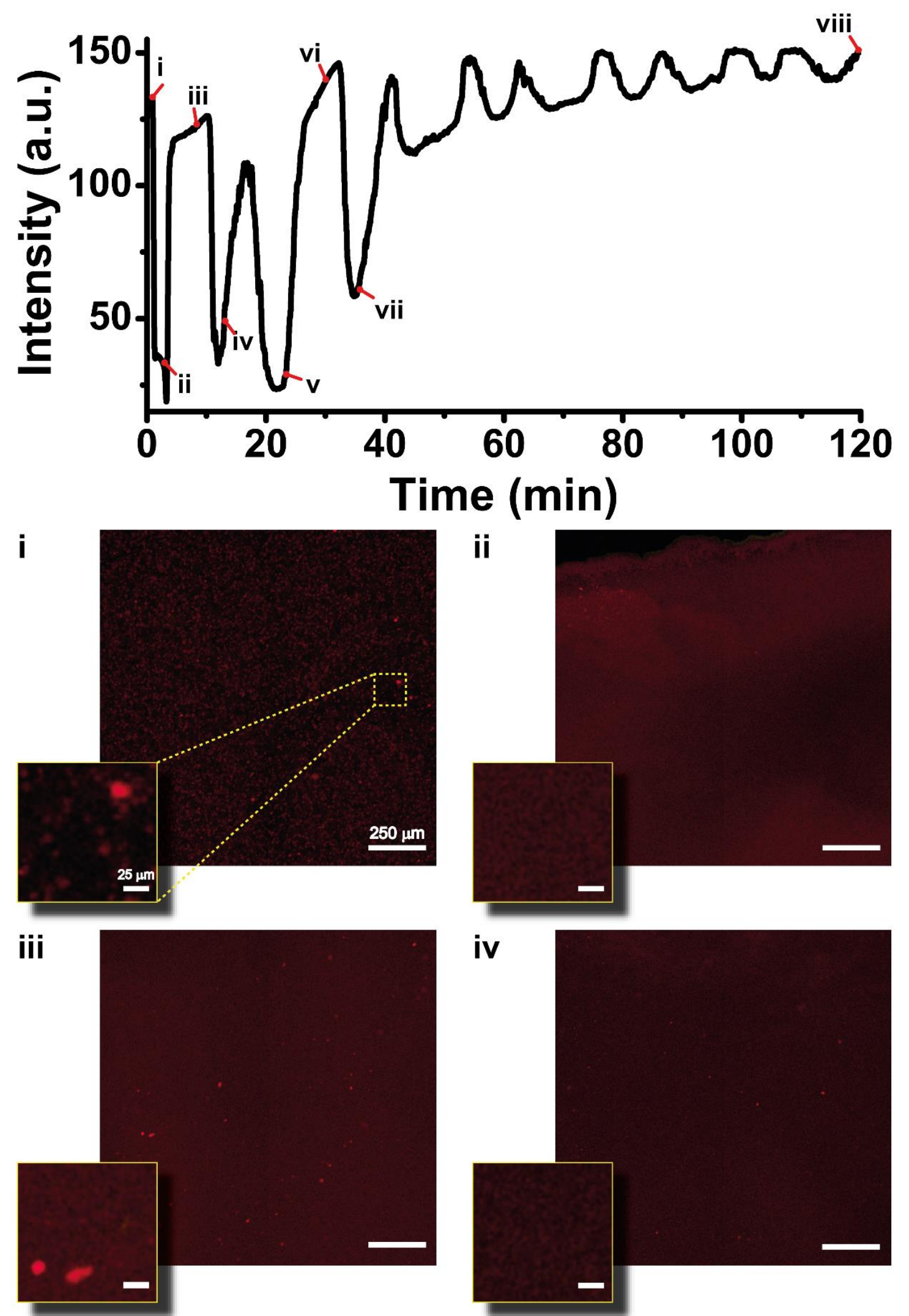

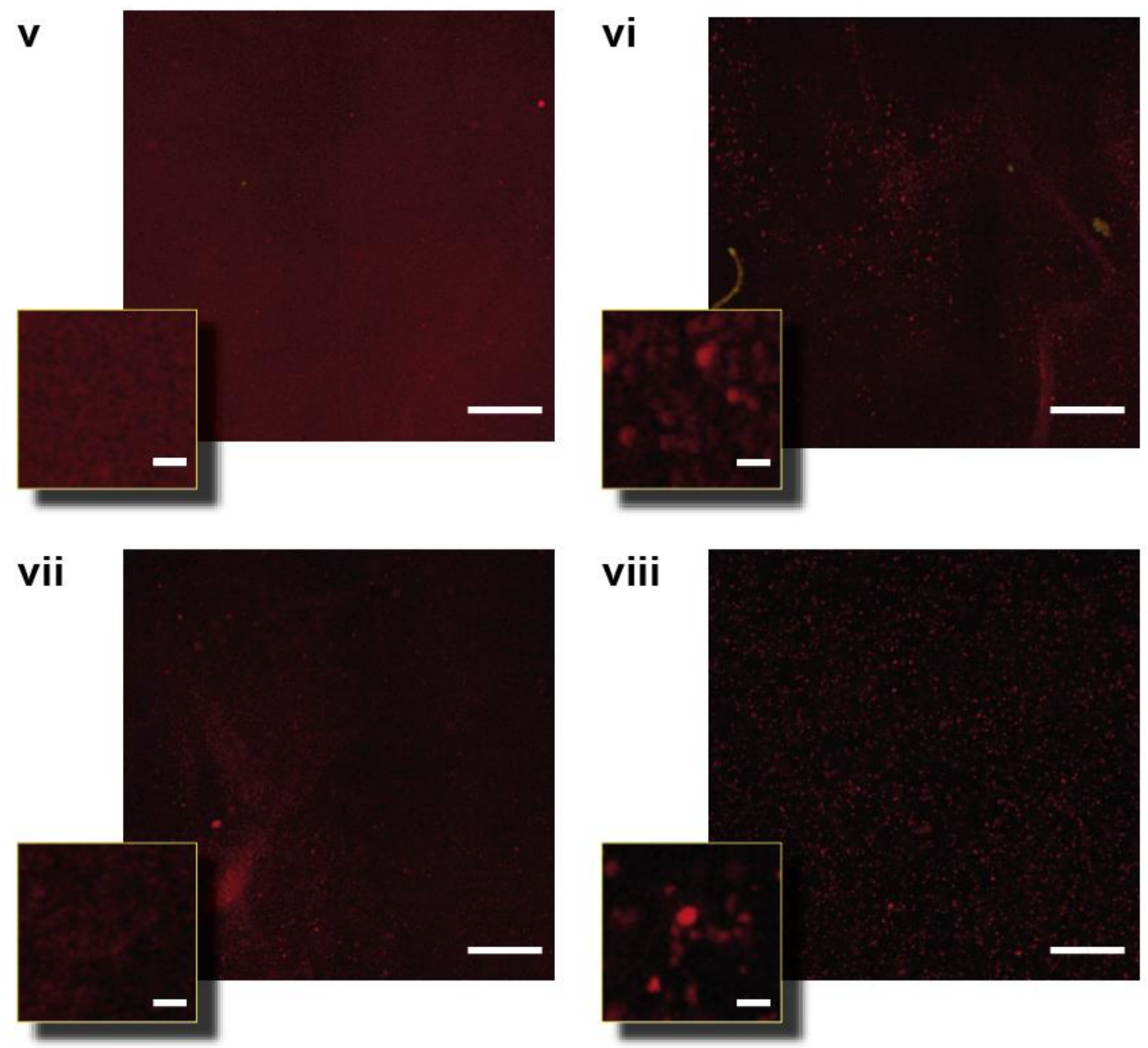

Supplementary Figure 13. Confocal micrographs during supramolecular oscillations. Aliquots from a $75 \mu \mathrm{M}$ PDI solution during oscillations (followed with a CCD colour camera, black line shows red channel intensity) were measured using a Zeiss LSM 710 confocal microscopy (see methods). Red emissive assemblies can be observed in a freshly prepared PDI solution (i, $t=0 \mathrm{~min}$ ). $\mathbf{P D I}^{2-}$ is formed after addition of $\mathrm{Na}_{2} \mathrm{~S}_{2} \mathrm{O}_{4}$ inducing complete disassembly at the resolution of the microscope (ii, $t=2 \mathrm{~min}$ ). Oxidation leads to PDImon which grows via cooperative polymerization. Red emissive assemblies can be observed when PDI $_{\text {assem }}$ are present in solution (iii, $t=10 \mathrm{~min}$ ). The assemblies mostly disappear during oscillations (iv, $t=$ $13 \mathrm{~min}, \mathrm{v}, t=23.3 \mathrm{~min}$, and vii, $t=35.6 \mathrm{~min}$ ). Micrograph viii ( $\mathrm{t}=120 \mathrm{~min}$ ) shows that the number of PDlassem stays consistently high in the damped region (after $\mathrm{t} \sim 60 \mathrm{~min}$ ). In general, only during the first 3-5 large scale oscillations do all of the PDIassem disappear. 

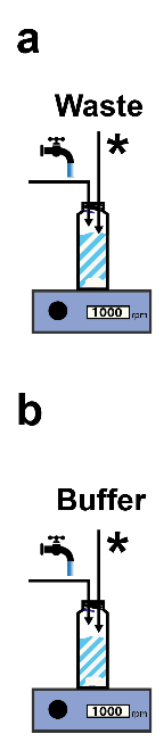
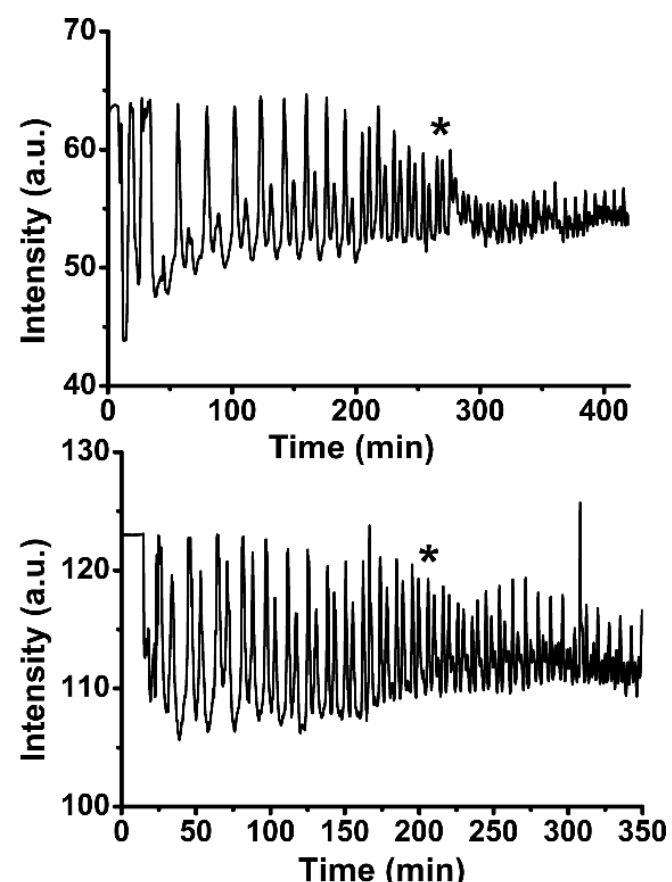

Supplementary Figure 14. Waste and buffer addition experiments during supramolecular oscillations. $2.25 \mathrm{~mL}$ of a $100 \mu \mathrm{M}$ PDI borate buffer solution was reduced to $\mathbf{P D I}^{2-}$ by adding $2 \mu \mathrm{L}$ of a 400 $\mathrm{mM} \mathrm{Na}_{2} \mathrm{~S}_{2} \mathrm{O}_{4}$ solution. Constant inflow of reductant $(0.7 \mu \mathrm{L} / \mathrm{min})$ was set once $\mathbf{P D I}_{\text {mon }}$ was in solution. a, 250 $\mu \mathrm{L}$ of a $400 \mathrm{mM}$ old dithionate solution (prepared the day before) was added after $250 \mathrm{~min}, \mathbf{b}, 250 \mu \mathrm{L}$ of buffer solution was added after $225 \mathrm{~min}$. These experiments show that neither waste accumulation nor volume changes significantly change the oscillations.

\section{Supplementary Section 5. Supramolecular oscillator model}

The model can be derived using basic nucleation-elongation-fragmentation kinetics. ${ }^{4}$ Continuation analysis and simulations of the model were performed with MatCont (downloadable from https://sourceforge.net/projects/matcont/). Simulations were done with the ODE45 routine provided by MatCont 5.1 within Matlab 8.4.0. The master equation for an ensemble of polymers is given by the time evolution of the concentrations $f(t, j)$, where $j$ indicates the length of the polymer and where we suppress the time dependence in the notation in the following:

$$
\begin{aligned}
& \frac{\partial f(j)}{\partial t}=2 m k_{e l} f(j-1)-2 m k_{e l} f(j)+2 k_{\text {red }} f(j-1)-2 k_{\text {red }} f(j)-f_{\text {frag }}(j-1) f(j)+ \\
& 2 k_{\text {frag }} \sum_{i=j+1}^{\infty} f(i)+k_{n u c} m^{n_{c}} \delta_{j, n_{c}}
\end{aligned}
$$

Depolymerisation is assumed to occur only in the reduction step to the $R$ type of monomers (vs monomers that are oxidised $O$, which can polymerize). No fusion (inverse of fragmentation) or secondary nucleation is considered here. The condition $f(j)=0$ for all $j<n_{c}$, where $n_{c} \geq 2$ is the critical nucleus size for the filament growth. From Eq. (1) we derive the kinetic equations for the number $P$ and mass concentrations $M$ :

$P(t)=\sum_{j} f(t, j)$ and $M(t)=\sum_{j} j \cdot f(t, j)$

By taking the sum over $j$ on both sides of Eq. (1). We obtain

$$
\begin{aligned}
& \frac{d[P]}{d t}=k_{\text {frag }}\left([M]-\left(2 n_{c}-1\right)[P]\right)+k_{\text {nuc }}[O]^{n_{c}} \\
& \frac{d[M]}{d t}=2\left(k_{e l}[O]-k_{\text {red }}-k_{\text {frag }} n_{c}\left(n_{c}-1\right) / 2\right)[P]+n_{c} k_{n u c}[O]^{n_{c}}
\end{aligned}
$$

where the contribution $k_{\text {red }} f\left(n_{c}\right)$ has been neglected to obtain a closed expression. The contribution of the dissociation of the nucleus to the overall dynamics is assumed to be small. The mathematical structure of the kinetic equations depends on the value of the critical nucleus size 
$n_{c}$. For simplicity, we fix $n_{c}=2$ for the remainder of this article (simulations not displayed here show that oscillations can also be found for $n_{c}=3,4$ ) and we obtain:

$\frac{d[P]}{d t}=-3 k_{f r a g}[P]+k_{f r a g}[M]+k_{n u c}[O]^{2}$

$\frac{d[M]}{d t}=-2\left(k_{\text {frag }}+k_{\text {red }}\right)[P]+2 k_{e l}[P][O]+2 k_{\text {nuc }}[O]^{2}$

The reduced type of monomers $R$ obeys the following mass-action expression

$\frac{d[R]}{d t}=k_{\text {red }}[P]-k_{o x}[R]$

and the overall mass conservation yields for the concentration of oxidized monomers $O$ (with $[\mathrm{m}]$ tot being the total mass):

$[O]=[m]_{t o t}-[M]-[R]$

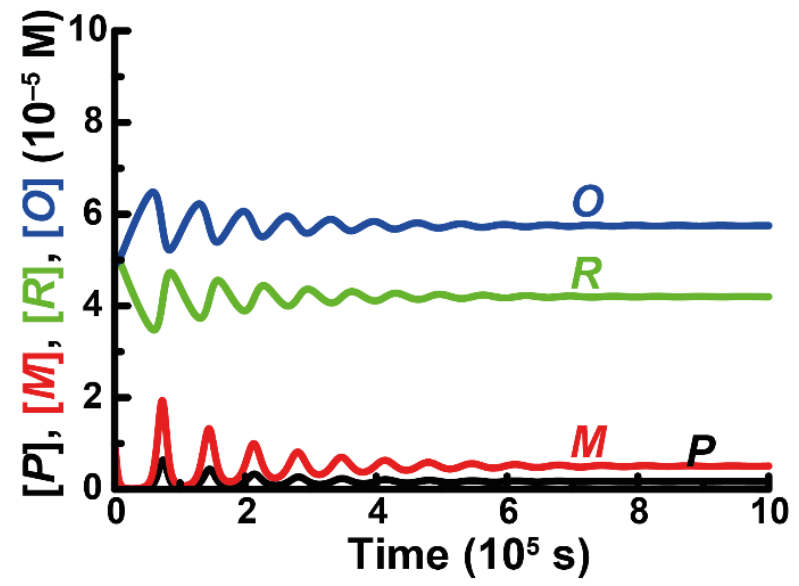

Supplementary Figure 15. Numerical simulations of oscillations in all species. All variables $(P, M, R$ and $O$ ) show oscillating behaviour. Initial conditions: $[P]=1 \cdot 10^{-8} \mathrm{M},[\mathrm{M}]=1 \cdot 10^{-7} \mathrm{M},[R]=5 \cdot 10^{-6} \mathrm{M},[\mathrm{m}]_{\text {tot }}=$ $1 \cdot 10^{-5} \mathrm{M}$; constants: $k_{n u c}=4 \cdot 10^{-4} \mathrm{M}^{-1} \mathrm{~s}^{-1}, k_{e l}=400 \mathrm{M}^{-1} \mathrm{~s}^{-1}, k_{\text {frag }}=3 \cdot 10^{-4} \mathrm{~s}^{-1}, k_{\text {red }}=1.5 \cdot 10^{-3} \mathrm{~s}^{-1}, k_{o x}=5 \cdot 10^{-}$ ${ }^{6} \mathrm{~s}^{-1}$.

\section{Supplementary Section 6 . Supramolecular fronts and patterns}

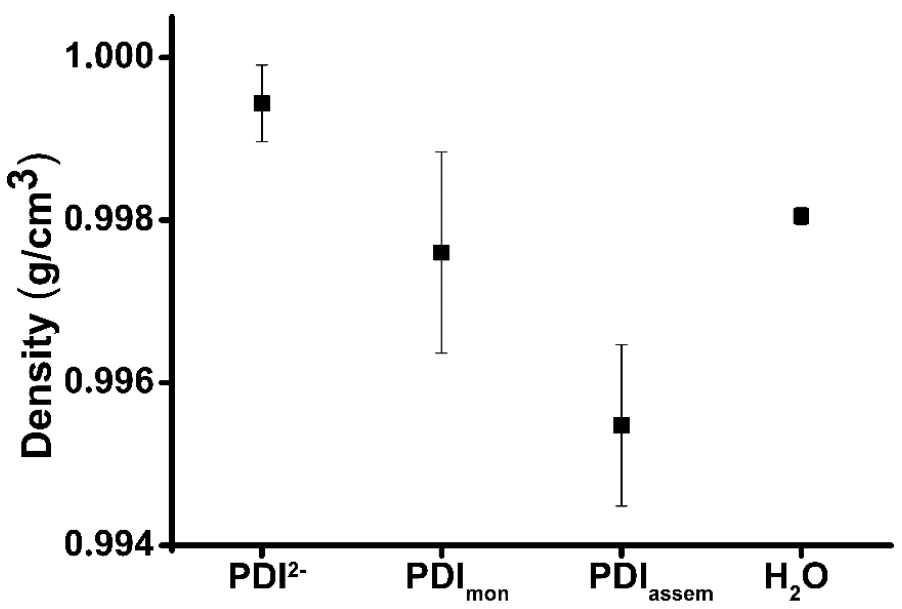

Supplementary Figure 16. Density measurements. $6 \mathrm{~mL}$ of a $100 \mu \mathrm{M}$ PDI borate buffer solution was reduced to $\mathbf{P D I}^{2-}$ by adding $4 \mu \mathrm{L}$ of a $400 \mathrm{mM} \mathrm{Na}_{2} \mathrm{~S}_{2} \mathrm{O}_{4}$ solution. The density of $\mathbf{P D I}^{2-}$ was measured.

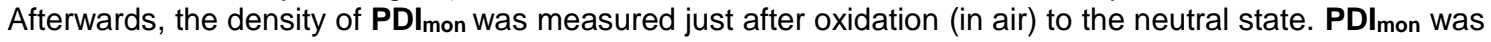
aged for $1 \mathrm{~h}$ leading to PDlassem. Water was measured as a control experiment. All measurements were repeated at least 4 times. The difference in density between $\mathbf{P D I}$ mon and $\mathbf{P D I} \mathbf{l}_{\text {assem }}$ is statistically significant, judged by the two-tailed $P$ value of $0.0363(P<0.05)$. 
a
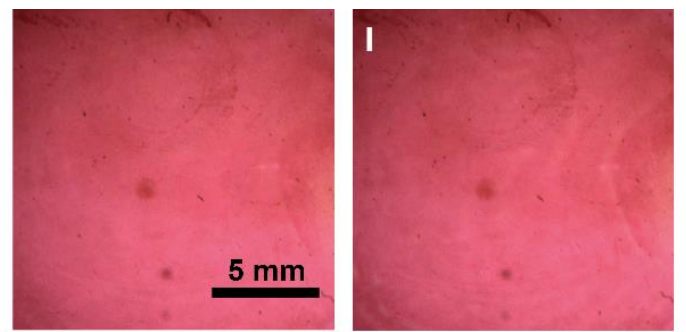

IV
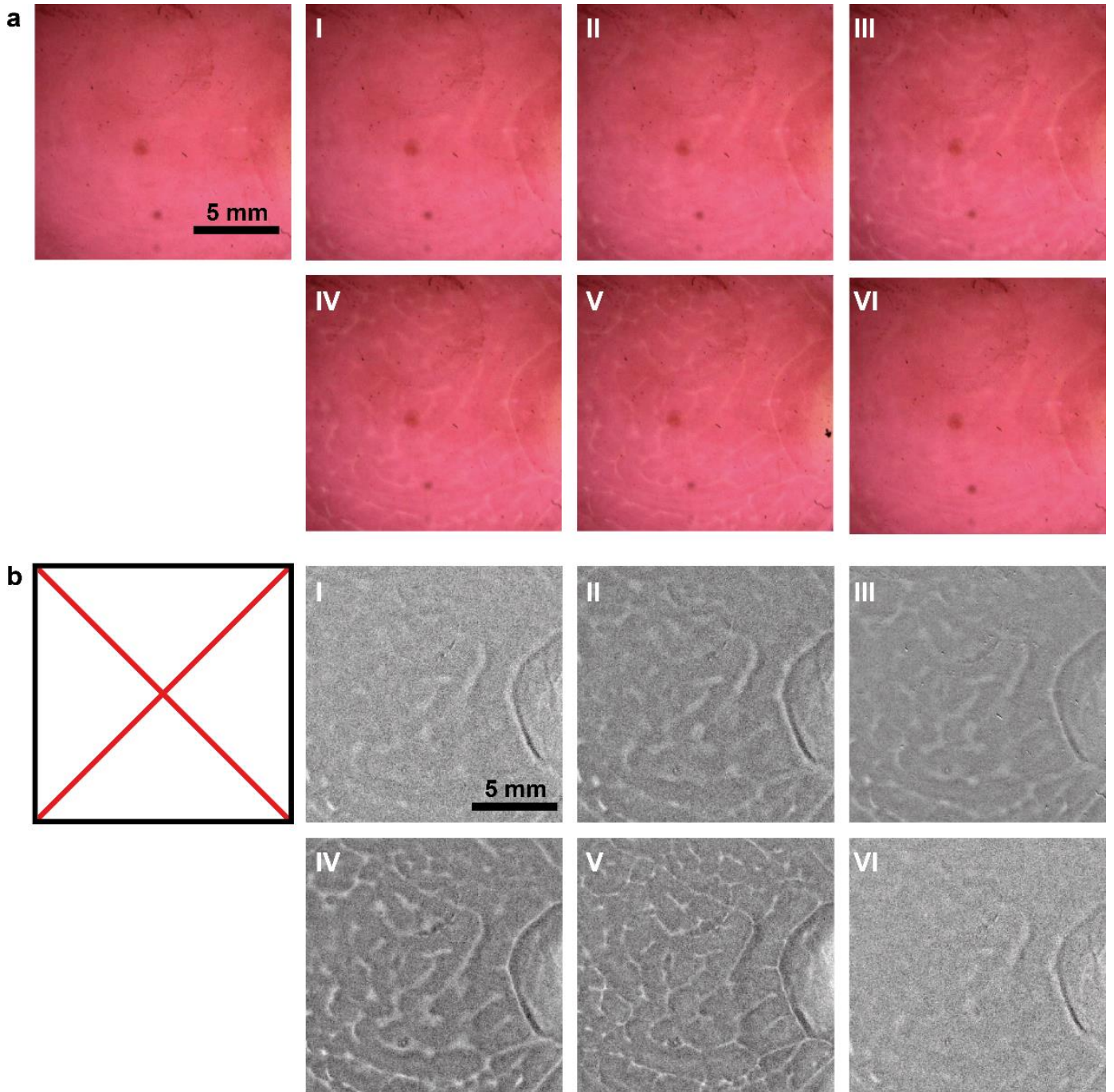

Supplementary Figure 17. Pattern formation. Transient formation of patterns (appearing after $30 \mathrm{~min}$ ) was observed in a round chip (diameter $12.5 \mathrm{x}$ height $1 \mathrm{~mm}$ ) while performing propagating front experiments. a, Real colour snapshots, $\mathbf{b}$, previous snapshots were background corrected by dividing by the initial image (i.e., top left in panel a). Times of the snap shots are: i) $30 \mathrm{~min}$, ii) $33 \mathrm{~min}$, iii) $36 \mathrm{~min}$, iv) $39 \mathrm{~min}$, v) $42 \mathrm{~min}$, vi) $45 \mathrm{~min}$. 


\section{Supplementary Section 7. Captions for videos}

Supplementary video 1. Supramolecular oscillations in a semi-batch reactor. A PDI mon $(2.25$ $\mathrm{mL})$ solution $(75 \mu \mathrm{M})$ was recorded under continuous influx of a $400 \mathrm{mM} \mathrm{Na}_{2} \mathrm{~S}_{2} \mathrm{O}_{4}$ solution $(0.7$ $\mu \mathrm{L} / \mathrm{min}$ ) at constant stirring rate (i.e., $1000 \mathrm{rpm}$ ) in a well-ventilated box (constant oxygen supply and light). Colour changes can be observed during supramolecular oscillations between the different assembled (turbid pink colour) and disassembled (clear purple colour) states.

Supplementary video 2. Propagating front of supramolecular assemblies. A freshly oxidized PDI $_{\text {mon }}$ solution $(1.5 \mathrm{~mL}$ of a $100 \mu \mathrm{M}$ PDI solution) was immediately injected into the 3D printed chip $(50 \times 25 \times 1 \mathrm{~mm})$. A propagating front in adjacent volumes was observed immediately after addition of $\mathbf{P D I}$ assem seeds ( $30 \mu \mathrm{L}$ of a $1 \mathrm{~h}$ old $\mathbf{P D I}$ mon solution) from one side of the chip. Images were acquired using a stereoscope (Nikon SMZ7457) equipped with a CCD camera, frames were recorded every 10 seconds for 60 min. The data shown in Fig. 4b,c corresponds to the video.

Supplementary video 3 . The propagating front induces large scale in-plane convection. Large-scale in plane convection was observed in a propagating front experiment. Images were acquired using a ZEISS LSM 710 confocal microscope, frames were recorded every 2 seconds for $30 \mathrm{~min}$. The arrows indicate the direction of the flow and the length is proportional to the speed. The direction and velocity of the flow was tracked using PIVlab tool from Matlab.

Supplementary video 4. Control experiment. Seeded front vs. non-seeded region. A 3D printed chip $(45 \times 30 \mathrm{~mm})$ was used, which was covered by mineral oil. Buffer solution was added

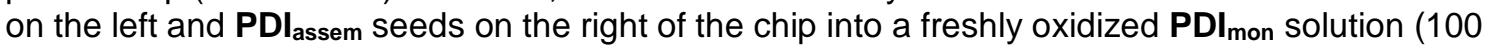
$\mu \mathrm{M}$, see methods for preparation). Images were acquired using a stereoscope (Nikon SMZ7457) equipped with a CCD camera, frames were recorded every 10 seconds for $50 \mathrm{~min}$.

Supplementary video 5 . An outward propagating oxidation front induces flow-alignment of

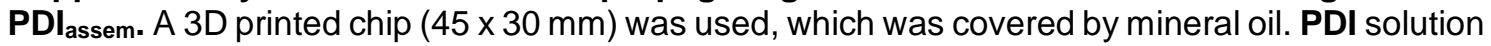
(1.5 mL, filling $3 \mathrm{~mm}$ height in the chip) was reduced to PDI ${ }^{2-}$ by adding $4 \mu \mathrm{L}$ of $400 \mathrm{mM} \mathrm{Na}_{2} \mathrm{~S}_{2} \mathrm{O}_{4}$ solution. PDI $^{2-}$ solution was poured into the chip and carefully covered with mineral oil. An outward moving propagating oxidation front and convection through the entire cell was observed. In addition, PDI assem structures aligned along the flow lines. Images were acquired using a stereoscope (Nikon SMZ7457) equipped with a CCD camera, frames were recorded every 10 seconds for $100 \mathrm{~min}$. The data shown in Fig. $4 \mathrm{~d}$ corresponds to the video. 


\section{Supplementary References}

1. Leira-Iglesias, J., Sorrenti, A., Sato, A., A. Dunne, P. \& M. Hermans, T. Supramolecular pathway selection of perylenediimides mediated by chemical fuels. Chem. Commun. 52, 90099012 (2016).

2. Kulkarni, C., Meijer, E. W. \& Palmans, A. R. A. Cooperativity Scale: A Structure-Mechanism Correlation in the Self-Assembly of Benzene-1,3,5-tricarboxamides. Acc. Chem. Res. 50, 19281936 (2017).

3. Meisl, G. et al. Molecular mechanisms of protein aggregation from global fitting of kinetic models. Nat. Protoc. 11, 252-272 (2016).

4. Cohen, S. I. A. et al. Nucleated polymerization with secondary pathways. I. Time evolution of the principal moments. J. Chem. Phys. 135, 065105 (2011). 